\title{
Model-Based Control and Optimization of Large Scale Physical Systems - Challenges in Reservoir Engineering
}

\author{
Paul M.J. Van den Hof $^{1}$, Jan-Dirk Jansen ${ }^{2,3}$, Gijs van Essen ${ }^{2,1}$, Okko H. Bosgra ${ }^{1}$ \\ 1. Delft Center for Systems and Control, Delft University of Technology, Mekelweg 2, 2628 CD Delft, The Netherlands \\ E-mail: p.m.j.vandenhof@tudelft.nl \\ 2. Department of Geotechnology, Delft University of Technology, The Netherlands \\ E-mail: j.d.jansen@tudelft.nl \\ 3. Shell International Exploration and Production, Rijswijk, The Netherlands
}

\begin{abstract}
Due to urgent needs to increase efficiency in oil recovery from subsurface reservoirs new technology is developed that allows more detailed sensing and actuation of multiphase flow properties in oil reservoirs. One of the examples is the controlled injection of water through injection wells with the purpose to displace the oil in an appropriate direction. This technology enables the application of model-based optimization and control techniques to optimize production over the entire production period of a reservoir, which can be around 25 years. Large scale reservoir flow models are used for optimizing production settings, but suffer from high levels of uncertainty and limited validation options. One of the challenges is the development of reduced complexity models that deliver accurate long-term predictions, and at the same time are not more complex than can be warranted by the amount of data that is available. In this paper an overview will be given of the problems and opportunities for model-based control and optimization in this field aiming at the development of a closed-loop reservoir management system.
\end{abstract}

Key Words: Oil reservoir, production optimization, optimal control, parameter estimation, dynamic optimization, identifiability, nonlinear observers

\section{INTRODUCTION}

The production of oil and gas from petroleum reservoirs is a typical example of a large scale dynamical system where complex physics governs the (nonlinear) dynamics of the underlying models, and operations are classically driven by decisions based on operator experience and supported by scenario studies. Given the economic importance to optimize the depletion efficiency of the reservoirs there is a serious challenge to rationalize the decision processes. With newly developed technology for drilling wells and instrumenting wells with actuators and sensors, there is an increasing opportunity for model-based control and optimization to develop rational and model-based decision support systems so as to optimize the economic efficiency of the process $[19,15]$. However the size and complexity of the underlying systems, raise additional challenges for the systems and control tools that can be applied.

Oil and gas reside inside the pores of subsurface rock layers. From above the oil and gas-baring layers are sealed by an impermeable layer of rock stopping the oil and gas from migrating to the surface. In some cases, a water bearing layer - referred to as an aquifer - is present below the oil and gas-bearing layer that provides pressure support during production of oil and gas. Such a closed off subsurface environment containing oil, water and gas is referred to as a petroleum or oil reservoir.

This work is supported by Senter/Novem and Shell in the scope of the project VALUE, and by Shell and TNO in the scope of the project ISAPP.
The production life of a petroleum reservoir generally lasts a number of decades and usually two or three production phases can be identified. In the primary production phase wells are drilled from the surface into the reservoir. The over-pressurized reservoir provides the driving force to push the oil to the surface. Although this process requires no additional effort than drilling wells, unfortunately only $5 \%-15 \%$ of the total amount of oil can be recovered in this manner. When the reservoir pressure decreases, the production rates drop as well. To maintain a preferred production level, the application of an external force is required. This is generally done by either injecting fluids (water or gas) into the reservoir or by installing pumps. Somewhere between $20 \%-70 \%$ of the oil can be recovered using secondary production methods. Tertiary production methods are aimed at changing the properties of the fluids (oil, gas or water) to improve recovery, as e.g. injecting steam, adding polymers to injected water or by injecting surfactants. With these methods up to $90 \%$ of the oil can be recovered.

Although a recovery factor of up to $90 \%$ can theoretically be reached going through the three production phases, the economical threshold generally lays around $35 \%$. This is partly due to the relatively large costs associated with secondary and tertiary methods, but it is also the result of an inefficient deployment of these methods, due to a lack of knowledge how to operate these phases in a structured and economically efficient way. Rather than operating on the basis of operator experience, there are opportunities to optimize the production by model-based control and optimiza- 
tion, on the basis of predictions of mathematical reservoir models.

In this paper we will give an overview of the challenges and opportunities for model-based control and optimization in this domain of technology. Our aim is to improve economic performance over the production life of the reservoir. We will only focus on the secondary production method of $w a-$ terflooding, which involves the controlled injection of water into the reservoir. Waterflooding is selected as central depletion technique, because

- It is a very common and much applied technique.

- Reservoir models can give relatively accurate longterm predictions [2].

- The scope for improvement compared to current practice is significant.

- Many input/decision variables are available providing the necessary degrees of freedom for applying (model-based) control.

The paper proceeds as follows. In Section 2 the process of waterflooding is explained in further detail. The (general) reservoir model is discussed in Section 3. In Section 4, perspectives on control and optimization of economic lifecycle performance of oil production are discussed. Nominal optimization of the problem in which the role of uncertainty is neglected is presented in Section 5. Section 6 discusses how the optimal control problem can be attacked when uncertainty is present. State and parameter estimation is the subject of Section 7. An example of a closedloop approach is presented in Section 8. Section 9 deals with the estimation and identifiability of model parameters. In Section 11, some additional opportunities for the field of system and control to contribute to improved oil recovery are presented. Finally, the paper is concluded in Section 12.

\section{WATERFLOODING}

Waterflooding is the most popular secondary recovery method. To get an idea, over $50 \%$ of USA oil production is due to waterflooding. The goal of the method is to maintain or increase reservoir pressure to boost production and to displace oil from the pores of the reservoir rock and replace it by water.

Oil is produced through production wells through which the oil flows to the surface. For the injection of water particular injection wells are drilled. Over the recent years, increasingly more so-called smart or intelligent wells are drilled. These smart wells are equipped with multiple downhole variable control valves, each for a different section of the well. Using these valves, the amount of water injected into and oil produced from a specific geological layer can also be controlled. These smart wells vastly expand the degrees of freedom (DOF) for control.

The injected water travels through the reservoir, away from the injection wells, but generally not in a uniform manner. This is the result of the strong heterogeneous nature

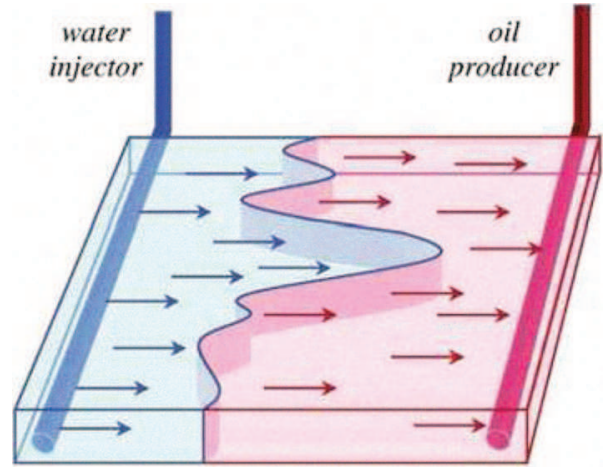

Figure 1: Waterflooding of a schematic $2 D$ reservoir with a horizontal injection and production well, both with spatially distributed control valves. Heterogeneity of the reservoir causes fingering effects in the oil-water front.

of reservoir rock. As a result, the oil-water front shows so-called fingering effects: in certain parts of the reservoir the fluids experience less resistance and locally the front moves much faster. This fingering effect can also be observed in the schematic Figure 1, where water is injected into a $2 \mathrm{D}$ reservoir using a horizontal injection well and oil is produced using a horizontal production well. Both wells have spatially distributed control valves. The block diagram in Figure 2 shows (schematically) the multiple actuation inputs and measurement outputs available to control the water flooding process.

When in a particular region the oil-water front reaches a production well, water is produced and the production well is closed (shut in). Then the production capacity of this well inlet is lost, and oil that is left in the reservoir may have been by-passed. The challenge of control here is to dynamically manipulate the spatially distributed injector and producer valves so as to achieve maximum oil production over the lifetime of the reservoir.

\section{MODELLING}

The dynamics of the waterflooding process is described by a so-called reservoir model based on conservation of mass and momentum equations to describe the flow of oil, water or gas through the reservoir rock [2]. For reasons of simplicity we limit attention here to reservoirs with only oil and water present.

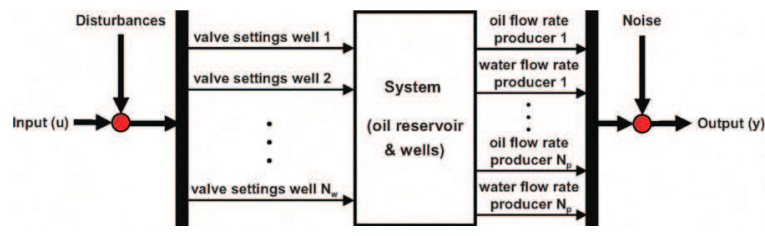

Figure 2: Block diagram of the oil reservoir as system. The inputs involve the valve settings of all $N_{w}$ wells. The outputs are the flow rate of oil and water for all the $N_{p}$ production wells 


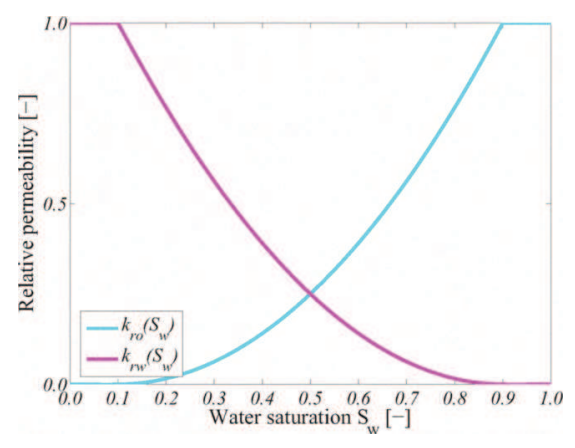

Figure 3: Relative permeability curves of oil $k_{\text {ro }}$ and water $k_{r w}$ as a function of the water saturation $S_{w}$.

The mass balance is expressed as follows:

$$
\nabla\left(\rho_{i} u_{i}\right)+\frac{\partial}{\partial t}\left(\phi \rho_{i} S_{i}\right)=0, \quad i=o, w
$$

where $t$ is time, $\nabla$ the divergence operator $\left(\frac{\partial}{\partial x}+\frac{\partial}{\partial y}+\frac{\partial}{\partial z}\right)$, $\phi$ is the porosity (volume fraction of void space), $\rho_{i}$ is the density of the phase $i, u_{i}$ the superficial velocity, $S_{i}$ the saturation, defined as the proportion of the pore space occupied by phase $i$, where $o$ relates to the oil phase and $w$ to the water phase.

Conservation of momentum is governed by the NavierStokes equations, but is normally simplified for low velocity flow through porous materials, to be described by the semi-empirical Darcy's equation as follows (discarding gravity):

$$
u_{i}=-k \frac{k_{r i}}{\mu_{i}} \nabla p_{i}, \quad i=o, w,
$$

where $p_{i}$ is the pressure of phase $i, k$ is the absolute permeability, $k_{r i}$ is the relative permeability and $\mu_{i}$ is the viscosity of phase $i$. The permeability $k$ is an inverse measure of the resistance a fluid experiences flowing through the porous medium. The relative permeability $k_{r i}$ relates to the additional resistance phase $i$ experiences when other phases are present, due to differences in viscosity. This relationship is non-linear as can be observed in Figure 3, where the relative permeabilities $k_{r o}$ and $k_{r w}$ are plotted against the water saturation $S_{w}$. As a result, the reservoir model becomes strongly non-linear.

Substituting (2) into (1) results into 2 flow equations with 4 unknowns, $p_{o}, p_{w}, S_{o}$ and $S_{w}$. Two additional equations are required to complete the system description. The first is the closure equation requiring that the sum of phase saturations must equal 1:

$$
S_{o}+S_{w}=1
$$

Second, the relation between the individual phase pressures is given by the capillary pressure equation:

$$
p_{\text {cow }}=p_{o}-p_{w}=f_{\text {cow }}\left(S_{w}\right) \text {. }
$$

Common practice in reservoir simulation is to substitute (3) and (4) into the flow equations, by taking the oil pressure $p_{o}$ and water saturation $S_{w}$ as primary state variables:

$$
\begin{array}{r}
\nabla\left(\tilde{\lambda}_{o} \nabla p_{o}\right)=\frac{\partial}{\partial t}\left(\phi \rho_{o} \cdot\left[1-S_{w}\right]\right), \\
\nabla\left(\tilde{\lambda}_{w} \nabla p_{o}-\tilde{\lambda}_{w} \frac{\partial p_{c o w}}{\partial S_{w}} \nabla S_{w}\right)=\frac{\partial}{\partial t}\left(\phi \rho_{w} S_{w}\right),
\end{array}
$$

where $\tilde{\lambda}_{o}=k \frac{k_{r o}}{\mu_{o}}$ and $\tilde{\lambda}_{w}=k \frac{k_{r w}}{\mu_{w}}$ are the oil and water mobilities. Flow equations (5) and (6) are defined over the entire volume of the reservoir. It is assumed that there is no flow across the boundaries of the reservoir geometry over which (5)-(6) is defined (Neumann boundary conditions).

The model equations (5)-(6) are discretized in space, leading to a system built up of a finite number of blocks, referred to as grid blocks. After discretization in time also the following state space form results:

$$
V\left(x_{k}\right) \cdot x_{k+1}=T\left(x_{k}\right) \cdot x_{k}+q_{k}, \quad x_{0}=\bar{x}_{0},
$$

where $k$ is the time index and $x$ is the state vector containing the oil pressures $\left(p_{o}\right)$ and water saturations $\left(S_{w}\right)$ in all grid blocks. Vector $\bar{x}_{0}$ contains the initial conditions, which are assumed to be known. In the discretization of (5)-(6), the units are converted from $\left[\frac{\mathrm{kg}}{\mathrm{m}^{3} \mathrm{~s}}\right]$ to $\left[\frac{\mathrm{m}^{3}}{\mathrm{~s}}\right]$. In (7) a source vector $q_{k}$ is added to model the influence of the wells on the dynamic behavior of the reservoir. The source terms are usually represented by a so-called well model, which relates the source term to the pressure difference between the well and grid block pressure:

$$
q_{k}^{j}=w^{j} \cdot\left(p_{b h, k}^{j}-p_{k}^{j}\right),
$$

where $p_{b h, k}$ is the well's bottom hole pressure, $j$ the index of the grid block containing the well and $p_{k}^{j}$ the grid block pressure in which the well is located. The term $w$ is a well constant which contains the well's geometric factors and the rock and fluid properties of the reservoir directly around the well.

The geological properties inside each grid block are assumed to be constant. The strongly heterogeneous nature of the reservoir can be characterized by assigning different property values (most often the permeability) to each of the grid blocks. Usually a very large number of grid-blocks is required $\left(10^{2}-10^{6}\right)$ to adequately describe the fluid dynamics of a real petroleum reservoir.

Summarizing, reservoir models have the following properties:

- They generally have a MIMO structure. The number of wells may range from approximately 10 to 1000 .

- They are non-linear due to relative permeability effects.

- They are of a very high order due to spatial discretization.

\section{PERSPECTIVES FOR CONTROL AND OP- TIMIZATION}

The success of the oil production process is evaluated at the end of the production life of a reservoir. During its 
production life, the reservoir is never in steady-state. The waterflooding process can therefore be regarded as a batch process to which an optimal control strategy can be applied. As an economic cost function we use cumulative cash flow, where revenues are assigned to every barrel of oil produced and costs are assigned to every barrel of water either injected or produced. Additionally a annual discount factor is applied to weigh revenues over time. The discounted cumulative cash flow is referred to as Net Present Value (NPV) and can mathematically be represented by:

$J_{K}=\sum_{k=1}^{K}\left[\frac{r_{o} \cdot q_{o, k}-r_{w} \cdot q_{w, k}-r_{i n j} \cdot q_{i n j, k}}{(1+b)^{\frac{t_{k}}{\tau_{t}}}} \cdot \Delta t_{k}\right]$

where $r_{o}$ is the oil revenue $\left[\frac{\$}{m^{3}}\right], r_{w}$ the water production costs $\left[\frac{\$}{m^{3}}\right]$ and $r_{i n j}$ the water injection costs $\left[\frac{\$}{m^{3}}\right]$, which are all assumed constant. $K$ represents the total number of time steps $k$ of a fixed time span and $\Delta t_{k}$ the time interval of time step $k$ in $[d a y]$. The term $b$ represents the discount rate for a certain reference time $\tau_{t}$. The terms $q_{o, k}, q_{w, k}$ and $q_{i n j, k}$ represent the total flow rate of respectively produced oil, produced water and injected water at time step $k$ in $\left[\frac{m^{3}}{d a y}\right]$.

The injection flow rates and production valves settings can be dynamically operated over the production life, and therefore serve as control inputs to optimize the NPV. The success of a model-based control strategy will to a large extent rely on the quality of the dynamic model. However this is in general only known with limited accuracy, due to the fact that only limited information about the subsurface can be subtracted from available reservoir measurements. Either the measurements are global in nature, but are very noisy (e.g. seismic surveys) or the measurements themselves are accurate, but are only valid for a very small part of the reservoir (e.g. drilling core samples). In industrial practice very often use is made of prior knowledge on geological structures and fluid properties from reservoir experts, but the uncertainty in these models remains substantial. As a result there is a high demand for reliable models with quantified uncertainty bounds that are supported by on-line measurements during the production phase of the reservoir.

Because of the nonlinear dynamics of the flow equations, capturing the physical structure of the model is essential for accurate long-term predictions of the NPV.

In this paper we will show two approaches to deal with the optimal control problem:

1. An open-loop optimal control strategy, based on an exact dynamic model. This step shows the feasibility and potential of a model-based control strategy for the considered problem;

2. A closed-loop optimal control strategy, based on a nonlinear state observer and a model of which the parameters are estimated online in a data assimilation step.

The two approaches are discussed in the subsequent sections.

\section{OPTIMAL TRAJECTORY DESIGN}

\subsection{Introduction}

On the basis of an available reservoir model an (open-loop) optimal control problem can be solved to find the optimal valve settings for optimizing of the NPV over the life time of the reservoir. Mathematically, the optimal control problem can be expressed as follows:

$$
\begin{gathered}
\max _{u} J_{K}(u), \\
\text { s.t. } x_{k+1}=f\left(x_{k}, u_{k}\right), k=1, \ldots, K, \quad x_{0}=\bar{x}_{0}, \\
g(x, u) \leq 0
\end{gathered}
$$

where $u$ is the input trajectory, $f$ represents the system equations as described in (7) and $\bar{x}_{0}$ is a vector containing the initial conditions of the reservoir. The inequality constraints $g(x, u)$ relate to the capacity limitations of the wells.

For these large scale dynamic optimization problems, several approaches are available, as e.g. dynamic programming, simultaneous methods, multiple shooting methods and sequential methods [3]. The properties of reservoir models and the usually large number of control variables seriously limit the number of applicable dynamic optimization techniques.

Although other techniques might also become feasible for the considered types of problems, sequential, gradientbased optimization using an adjoint to calculate the gradients, is currently the main candidate for solving the very large oil recovery optimization problem. It was first introduced in petroleum literature for flooding optimization by Ramirez [19], but was ignored for quite some time because of the computational burden involved. It regained attention in the work of Brouwer [5] and Sarma [22], when the computational issues no longer caused any serious limitation of the method. The adjoint is now available in a number of the corporate and commercially available reservoir simulation packages, being also a major argument for considering this approach.

A vast number of gradient-based techniques exist that for the majority are based on applying Newton's method to the first order optimality conditions, e.g. SQP or generalized reduced gradient (GRG) methods [6, 4, 8]. However, in large regions within the decision variable space Net Present Value behaves linearly with changes of the decision variables, especially when far from an optimum. Within these regions, the second-order approximation is poor and the algorithms perform unsatisfactory. Therefore and for simplicity reasons, a steepest ascent algorithm is used:

$$
u_{i+1}=u_{i}+\tau \cdot \frac{\partial J}{\partial u}, \quad i=1, . ., N_{i t e r}
$$

where $N_{\text {iter }}$ is the number of iteration required to converge to an optimal input $u^{*}$.

Although gradient-based optimization techniques dominate in the literature on oil recovery optimization, alternative methods are gaining more attention [26]. 


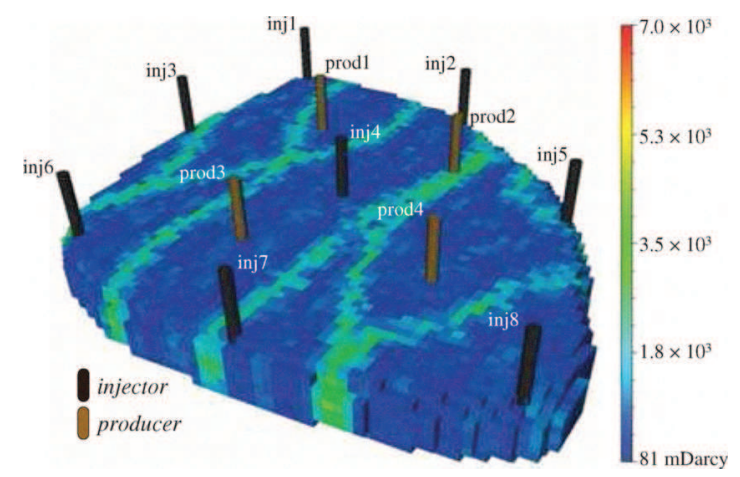

Figure 4: 3D reservoir model with 4 production and 8 injection wells. The geological structure involves a network of meandering channels in which the fluids flows experience less resistance, due to higher permeability.

\subsection{Nominal Optimization Example}

In order to illustrate the potential gain of a model-based control approach to the production optimization problem, the optimal control procedure is applied to a 3-dimensional oil reservoir model with 7 geological layers. The life-cycle of the reservoir covers a period of 8 years. The reservoir model consists of 18,553 grid blocks, as depicted in Figure 4 , and has dimensions of $480 \times 480 \times 28$ meter. Its geological structure involves a network of fossilized meandering channels in which the flowing fluids experience less resistance, due to higher permeability. The average reservoir pressure is 400 [bar].

The reservoir model contains 8 injection wells that inject water and 4 production wells. The production wells are modeled using a well model (8). In this experiment, it is assumed that the bottom hole pressures $p_{b h}$ can be controlled directly by the well operators. Of the injection wells, the injection flow rates can be manipulated. The minimum rate for each injection well is $0.0\left[\frac{\mathrm{m}^{3}}{d a y}\right]$, the maximum rate is set at a rate of $47.7\left[\frac{\mathrm{m}^{3}}{d a y}\right]$. The minimal bottom hole pressure $p_{b h}$ for each production well is $3827 \mathrm{kPa}$.

The objective function is the Net Present Value as shown in Eq. (9). The oil price is set at $283\left[\frac{\$}{m^{3}}\right]$, the water production (separation) costs at $31.4\left[\frac{\$}{\mathrm{~m}^{3}}\right]$ and no water injection costs at $\left(r_{i n j}=0\left[\frac{\$}{m^{3}}\right]\right)$. Furthermore, an annual discount factor is applied of $15 \%$, which is a frequently used discount factor in reservoir engineering.

The dynamic optimization procedure was executed as described in the previous section, using a system of adjoint equations to provide gradients of the objective function $J$ with respect to the injection rates $q_{i n j, k}$ and the bottom hole pressures $p_{b h}$ of the producers. Updates of the control $u$ were performed using a steepest ascent scheme (13).

The results of the dynamic optimization procedure are compared to a reactive control of the waterflooding project. Using reactive control, the injection wells inject continuously at their maximum capacity of $47.7\left[\frac{\mathrm{m}^{3}}{d a y}\right]$. The only

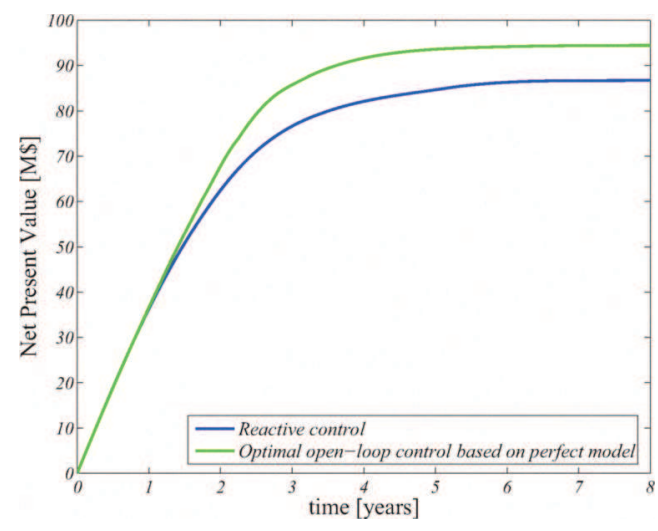

Figure 5: Net Present Value $J_{K}$ over time until $T_{f}=8$ years for the reactive control (dashed line) and the optimal control, optimized over 8 years, (solid line), determined using a perfect model.

control is active in the production wells, where the ratio between produced water and oil (watercut) is determined. If this ratio exceeds 0.9 , the production well is closed (shutin). This maximum watercut of $90 \%$ is an economic threshold and relates to the ratio between oil price and water production costs.

Figure 5 shows how the NPV $J_{K}$ evolves over time for the reactive control and the optimal control strategy. It can be observed that the benefits of applying the optimal control are considerable and the increase in NPV at the end of production life $\left(T_{f}\right)$ is $8.9 \%$. As a gradient-based optimization procedure is likely to find only a local optimum, this result may possibly improve when trying different initial values for the control input.

\section{CLOSED-LOOP DYNAMIC OPTIMIZA- TION}

In the open-loop dynamic optimization example, presented in the previous section, uncertainty and under-modeling effects were neglected. As a result, the example only shows that there is a potential scope to improve economic performance over the production life of a reservoir. The next challenge is how to realize this potential under more realistic conditions with the aid of available measurements.

Taking measurements during the production life of a reservoir is complex. This is the result of the tough conditions and limitations under which subsurface measurement devices must operate. Not surprisingly, these conditions and limitations form serious obstacles and until recently measurements were only taken at surface level. However, over the recent years much progress has been made. Today many more wells (smart wells) are equipped with downhole pressure gauges, flow meters and distributed temperature sensors. Besides these smart wells, time-lapse or $4 D$ seismic is gaining popularity, which is aimed at tracking the flooding process through several time-shifted seismic surveys. Although very noisy, these measurements provide information on regions of the reservoir where well measurement devices cannot. 


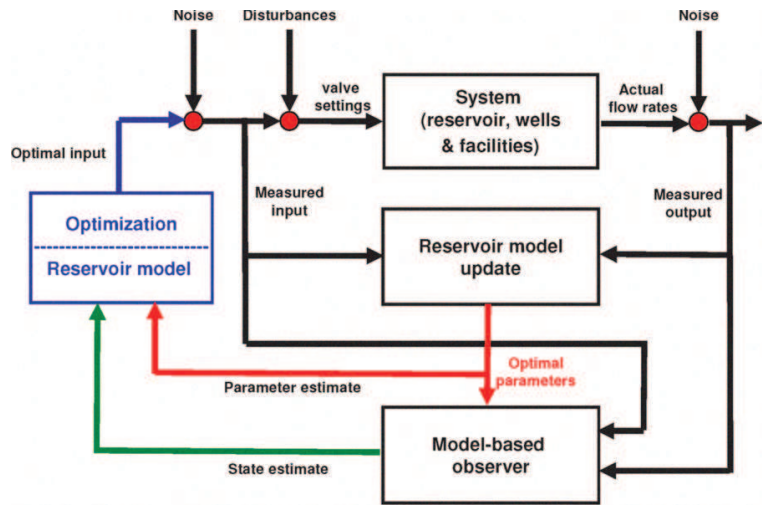

Figure 6: Closed-loop approach to improved reservoir management

Similar to the framework for (Nonlinear) MPC (NMPC) [1] a receding horizon approach can be followed to adjust the optimal control input on the basis of on-line measurement data. This takes place through the online estimation of an initial state through a nonlinear observer, as schematically indicated in Figure 6. In reservoir engineering this approach has first been adopted in the work of Brouwer [5]. It consists of the following steps:

- Based on a deterministic non-linear model with known initial conditions $x_{0}$, an optimal control strategy $u_{1}^{*}(t)$ is calculated from $t=t_{0}$ until $t=T_{f}$, where $t_{0}$ is the time at start production and $T_{f}$ is the end of the production life of the reservoir.

- The optimal waterflooding strategy is implemented for a horizon from $t=t_{0}$ until $t=t_{0}+T$, where $T$ is a time horizon for which $T<T_{f}-t_{0}$ holds.

- During the interval from $t=t_{0}$ until $t=t_{0}+T$, (subsurface) production measurements $y(t)$ are taken from the reservoir.

- Measurement data $y(t)$ and valve settings are used to estimate the state vector $\hat{x}_{0}$ at time $t_{0}+T$, and optionally to improve the estimate of the model parameters $\hat{\theta}$. This step is often referred to as data-assimilation.

- The improved model is used within the dynamic optimization step to calculate an improved control strategy $u_{2}^{*}(t)$ for the remaining horizon from $t=t_{0}+T=$ $t_{1}$ until $t=T_{f}$.

- The loop is executed until $t=T_{f}$ is reached.

Because of the batch-type nature of the reservoir process, the optimization strategy becomes a shrinking horizon approach. This has repercussions on the computational burden of the method; in the initial phase of the production relatively long prediction horizons have to be taken into account. Fortunately, the waterflooding process evolves very slowly and as a result the computational burden involved is generally not a problem in realistic circumstances.
When the data-assimilation step is solely aimed at estimating the states $x$, it is essentially an observer. Alternatively (or simultaneously), the model parameters $\theta$ may also be estimated. For reservoir models, the challenge of both state as parameter estimation is in the size of the model, its nonlinear properties and the fact that only limited and noisy measurements are available.

\section{STATE AND PARAMETER ESTIMATION}

In order to apply a receding/shrinking horizon principle to the optimal control problem, a state observer is necessary to estimate the current state. This is being done on the basis of the (nonlinear) state space model, given by:

$$
\begin{aligned}
x_{k+1} & =f\left(x_{k}, u_{k}\right)+Q w_{k} \\
y_{k} & =g\left(x_{k}, u_{k}\right)+R v_{k}
\end{aligned}
$$

where $w$ and $v$ are assumed to be Gaussian white noise processes that are mutually uncorrelated and have unit variance.

The most common observer technique for these type of nonlinear models is the Extended Kalman Filter (EKF), that processes the measurement data on the basis of the linear Kalman Filter equations, thereby utilizing a locally linearized process model and assuming a Gaussian distribution of the state and output. In the reservoir engineering field a generalization of this approach has become common, named as Ensemble Kalman Filter (EnKF) $[10,11]$. Its popularity is due to the properties that

- It empirically analyses the a posteriori distributions of the state and the output, by Monte Carlo simulations, thereby relying less on linear approximations of the nonlinear model, and

- It is easily extendable to also estimate unknown parameters, starting from a (finite) set of prior parameter estimates.

The basic algorithm of an EnKF filter encompasses the following steps:

- As prior information an ensemble of initial states $\left\{\hat{x}_{k \mid k}\right\}$ is synthetically generated from a given distribution;

- By simulating every ensemble member through the model (..) corresponding ensembles $\left\{\hat{x}_{k+1 \mid k}\right\}$ and $\left\{\hat{y}_{k+1 \mid k}\right\}$ are generated, that are stored as columns of matrices $\hat{X}$ and $\hat{Y}$ respectively;

- The measurement update of a common Extended Kalman Filter is applied to every element of the ensemble, where the covariance matrices are replaced by sampled estimates from the ensembles generated in the previous step;

- The measurement update then becomes

$$
\hat{x}_{k+1 \mid k+1}=\hat{x}_{k+1 \mid k}+K_{k+1}\left[y_{k+1}-\hat{y}_{k+1 \mid k}\right]
$$


where $K_{k+1}$ is generated by applying the best linear unbiased estimate, given by

$$
K_{k+1}=\hat{X} \hat{Y}^{T} \cdot\left[\hat{Y} \hat{Y}^{T}+R\right]^{-1}
$$

- The result is a new ensemble $\left\{\hat{x}_{k+1 \mid k+1}\right\}$.

In the EnKF the analytical propagation of the error covariance matrix is replaced by a Monte Carlo approach, in which the covariance matrix is computed from an ensemble of models. The EnKF utilizes the availability of the reservoir simulation model and does not require a linearization of this model. At the same time it remains computationally feasible for large scale models, up to millions of states, as has been shown in several applications, both in reservoir engineering problems and in different fields, see.e.g. $[18,13]$.

When interpreting the state estimation problem as a constrained least squares problem, see e.g. [20], the EnKF can be interpreted as constructing an appproximate gradient of the optimization problem on the basis of ensemble estimate of the (cross)-covariance between state and output signals. The popularity of EnKF in this domain is also explained by the relative ease with which not only states but also parameters can be included in the estimation problem. Following a Bayesian approach the unknown parameters are added to the states, leading to an extended state vector $\bar{x}=\left[x^{T} \theta^{T}\right]^{T}$. This extended state is then estimated with an EnKF on the basis of an ensemble of $\bar{x}$. This implies that also an ensemble of prior parameter estimates is added to the problem as a source of prior information.

In reservoir engineering problems the EnKF observer (data-assimilation) algorithm is applied to models with number of state variable up the order of $10^{5}$. Since the permeability in each grid block is usually considered to be an unknown parameter, the dimension of $\theta$ amounts to the number of grid blocks, being of the same order as the number of states. As a result an extremely large number of variables has to be estimated from the available data.

\section{CLOSED-LOOP SIMULATION EXAMPLE}

\subsection{Description of the example}

This closed-loop dynamic optimization example discussed here was originally presented in Jansen [16]. The problem setting is identical to the example presented in Section 5.2, with the only difference that now the grid block permeabilities $k$ are assumed to be unknown, except in the grid blocks that contain wells. This situation is also encountered in reality when oil is produced from a reservoir on which no seismic surveys were conducted.

The closed-loop dynamic optimization procedure of Section 6 and a gradient-based optimization of the control using the adjoint was used to optimize the control input. The data-assimilation step is performed using the EnKF as described in the previous section, estimating both the states $( \pm 37,000$ variables) and all parameters $( \pm 18,500$ variables). As shown in Section 3, the states relate to the oil pressures $p_{o}$ and the water saturation values $S_{w}$ in all

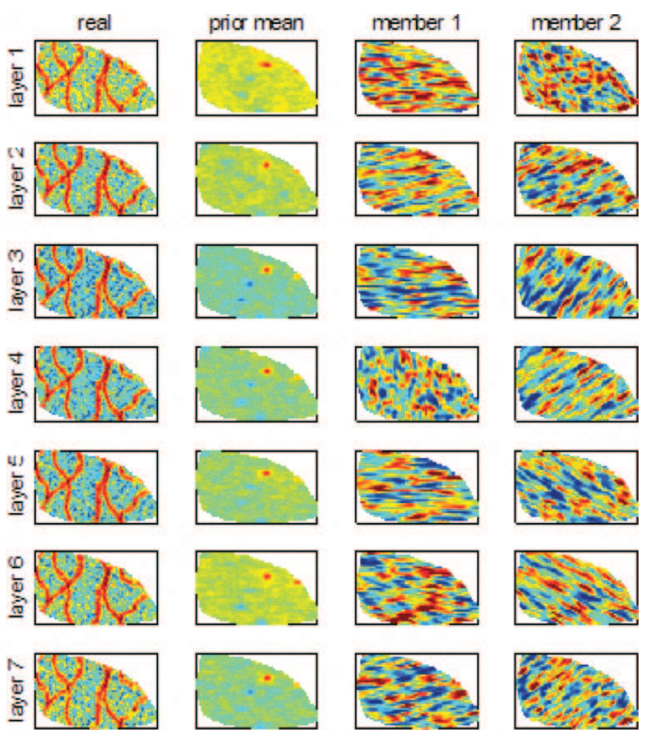

Figure 7: Seven layers of permeability of the real system (column 1), of the first 2 members of the ensemble with Gaussian random distribution (columns $3 \& 4$ ) and of the mean prior model over all 100 members (column 2).

grid blocks. The geological parameters $\theta$ that are updated involve the permeability values $k$ of each grid block. A model update is carried out on a yearly basis, i.e. $T=$ 365.25 [days].

The reservoir model containing the high permeability channels, displayed in Figure 4, serves as real system in this numerical experiment. Synthetic production data were generated using forward simulation of this system. This resulted in (synthetic) bottom hole pressure $\left(p_{b h, k}\right)$ and phase flow rate $\left(q_{o, k}\right.$ and $\left.q_{w, k}\right)$ measurements for all wells at 30-day intervals. To reflect realistic circumstances we added white Gaussian noise to the measured outputs $y$, having standard deviations for the oil rates, water rates and pressures of 15 $\left[\frac{m^{3}}{d a y}\right], 15\left[\frac{m^{3}}{d a y}\right]$ and $2800[k P a]$ respectively.

A set of 100 ensemble members was created using Gaussian random fields for permeability in each of the 7 layers, with a randomly-oriented ellipsoidal covariance with a randomly-chosen correlation length between 4 and 8 grid blocks. The members are conditioned to permeability of the grid blocks containing a well, which is assumed to be known. The first two members of the ensemble can be observed in Figure 7. The first column of Figure 7 shows the permeability field of the real system. It should be noted that its channel-like structure is not captured by the ensemble members. The mean permeability field over all prior ensemble members can be observed in the second column of Figure 7. For lack of a better estimate, the prior permeability field is assumed to be (more or less) homogeneous, except for the areas where wells are present. In this example, the mean permeability field was used in the gradientbased optimization of NPV. 

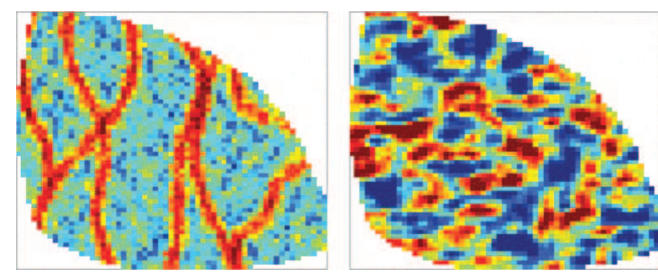

Figure 8: Single permeability layer of the real system (left) and of the final estimate of permeability (posterior) after execution of the closed-loop procedure.

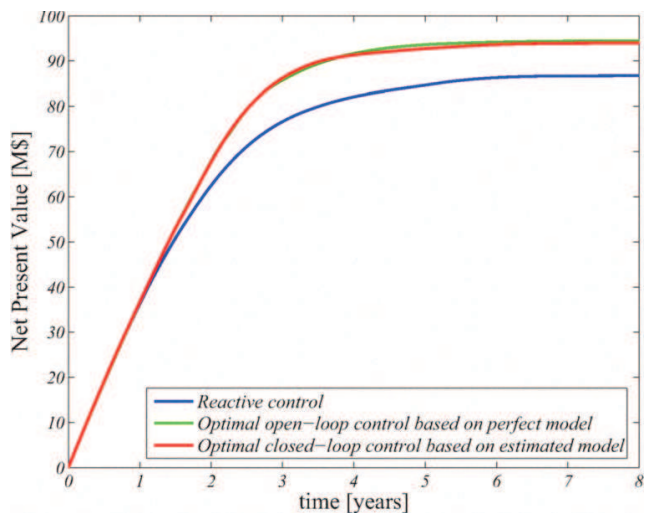

Figure 9: Net Present Value $J_{K}$ over time until $T_{f}=8$ years for the reactive control (blue), the optimal control, optimized over 8 years, for the ideal open-loop strategy (green) and the closed-loop strategy (red).

\subsection{Results}

The update of the model with estimated states and parameters is performed 7 times over the life of the reservoir. The final estimate(posterior) of a single layer of permeability can be observed in Figure 8, together with the real permeability field of that same layer. Based on a visual inspection of the results, the estimation of the permeability field seems poor. The results in NPV at $T_{f}$ are nonetheless quite good, as can be observed in Figure 9. The final NPV is 8.3\% higher than using the more conventional reactive strategy and is only $0.5 \%$ lower than the 'best' case scenario when the system is perfectly known.

\subsection{Conclusion}

The near-identical results in terms of NPV of the closedloop procedure is partly the result of the relative simplicity of the synthetic model. In real reservoirs, the wells are further apart and many more parameters are uncertain. However, still the issue remains that these different permeability fields lead to comparable results. This implies that (in this case) the essential information may be represented with a much less complex model. This also implies that there is room for reparameterization of the permeability field to reduce the number of model parameters, and for low-order modeling to reduce the number of states.

\section{ESTIMATING MODEL PARAMETERS AND IDENTIFIABILITY}

In this section we will consider the parameter estimation problem from a more principle point of view.

Due to the fact that grid block permeabilities are used to parametrize our model structure, the number of unknown parameters scales with the number of grid blocks that is used in the discretization of the underlying pde. As a direct consequence the number of parameters in the estimation problem becomes excessively large, and a relevant question becomes whether these parameters can be reliably estimated from measurement data. As a general rule of thumb, estimation of an overly large number of parameters leads to estimates with a very large variance, and even to lack of identifiability, i.e. parameter changes do not have an measurable effect on the output (dynamics) of the model [17]. We then conclude that the model is over-parametrized. The question then is, whether it is possible to reduce the physical parametrization to a level of complexity (i.e. a number of unknown parameters) that is warranted by the information content of the measurement data, and still maintains the physical interpretation that is necessary in order to make reliable long-term output predictions.

Consider the physics-based model structure

$$
\hat{\mathbf{y}}(\theta)=h\left(\mathbf{u}, \theta ; x_{0}\right),
$$

where $\hat{\mathbf{y}}$ is a prediction of $\mathbf{y}:=\left[\begin{array}{lll}y_{1}^{T} & \ldots & y_{N}^{T}\end{array}\right]^{T}$ denoting output signal measurements $y_{k} \in \mathbb{R}^{p}$ stacked over time, $\theta \in \Theta \subset \mathbb{R}^{q}$ the parameter vector, $\mathbf{u}:=$ $\left[\begin{array}{lll}u_{1}^{T} & \ldots & u_{N}^{T}\end{array}\right]^{T}$ the input vector $u_{k} \in \mathbb{R}^{m}$ stacked over time, and $x_{0}$ the initial state vector. We would now actually like to reduce the physics-base model structure $\hat{\mathbf{y}}=h\left(\mathbf{u}, \theta ; x_{0}\right)$ to an approximate structure in which the dimension of $\theta$ is considerably reduced, while the model structure itself does provide sufficient flexibility to accurately represent the input-output dynamics of the underlying system, in particular in those aspects where the dynamics influences the optimal control of the reservoir.

A global reparametrization of the model is hard as we simply do not know where to put the degrees of freedom (how to specify the dependence between the parameters). However in a local analysis appropriate tools can relatively easily be developed. The related notion of identifiability that we can investigate is formulated next ([12]):

Definition 1 An input/output model structure $h\left(\theta, \mathbf{u} ; x_{0}\right)$ : $\Theta \rightarrow \mathcal{H}$ is called locally identifiable in $\theta_{m} \in \Theta$ for a given $\mathbf{u}$ and $x_{0}$, if for all $\theta_{1}, \theta_{2}$ in the neighborhood of $\theta_{m}$ holds that $\left\{h\left(\mathbf{u}, \theta_{1} ; x_{0}\right)=h\left(\mathbf{u}, \theta_{2} ; x_{0}\right)\right\} \Rightarrow \theta_{1}=\theta_{2}$.

We consider a quadratic identification criterion in a prediction error setting [17], where the Hessian of the criterion provides a measure for the uniqueness of the parameter estimate, and thereby also as a sufficient condition for local identifiability under the given experimental conditions. The quadratic cost function is given by

$$
V(\theta):=\frac{1}{2} \epsilon(\theta)^{T} P_{v}^{-1} \epsilon(\theta),
$$


where the prediction error sequence $\epsilon$ is defined as

$$
\boldsymbol{\epsilon}(\theta)=\mathbf{y}-\hat{\mathbf{y}}(\theta)
$$

where $P_{v}$ is a weighting matrix that could represent (an estimate of) the covariance matrix of the noise sequence $v$ that is supposed to act on the measured output.

After approximation of $\hat{\mathbf{y}}$ with a first -order Taylor expansion around $\hat{\theta}$, the Hessian of $V(\theta)$ with respect to the parameters is

$$
\frac{\partial^{2} V(\theta)}{\partial \theta^{2}}=\frac{\partial \hat{\mathbf{y}}(\theta)^{T}}{\partial \theta} P_{v}^{-1}\left(\frac{\partial \hat{\mathbf{y}}(\theta)^{T}}{\partial \theta}\right)^{T},
$$

while

$$
\operatorname{cov}(\hat{\theta})=J^{-1}
$$

with $J$ the Fisher information matrix

$$
J=\mathbb{E}\left[\left.\frac{\partial^{2} V(\theta)}{\partial \theta^{2}}\right|_{\hat{\theta}}\right]
$$

and $\mathbb{E}$ denotes expectation.

Local identifiability at $\hat{\theta}$ can now be investigated by evaluating the SVD of the Hessian that can be obtained from:

$$
\frac{\partial \hat{\mathbf{y}}(\theta)^{T}}{\partial \theta} P_{v}^{-\frac{1}{2}}=\left[\begin{array}{ll}
U_{1} & U_{2}
\end{array}\right]\left[\begin{array}{cc}
\Sigma_{1} & 0 \\
0 & \Sigma_{2}
\end{array}\right]\left[\begin{array}{l}
V_{1}^{T} \\
V_{2}^{T}
\end{array}\right]
$$

where the separation between $\Sigma_{1}$ and $\Sigma_{2}$ is chosen in such a way that the singular values in $\Sigma_{2}$ are considerably smaller than those in $\Sigma_{1}$.

If we now reparametrize the model structure by employing the reduced parameter $\rho$ determined by $\theta=U_{1} \rho$, we have realized a model structure approximation, in which the parameters to be identified are well identifiable with a limited variance and the physical interpretation of the parameters remains untouched. The singular vectors that occur as the columns in $U_{1}$ actually can be seen as basis functions in the parameter space [23].

In the Bayesian approach to the parameter estimation problem, as discussed in the EnKF approach of Section 7, the over-parametrization is circumvented by adding a regularization term, in effect an a priori information on the parameters to be estimated. A consequence of this is that in case of poor identifiability properties of the model structure, the final parameter estimate might be dominated by the prior information, rather than determined by the measurement data.

\section{APPROXIMATING MODELS}

The results of the closed-loop example, presented in Section 8 , suggest that the essential dynamics of the flooding process may be represented with a much less complex model and a reduced number of parameters. This assumption is supported by the results of Heijn et al. and Zandvliet et al. $[14,28]$, where the controllability and observability aspects of the model were investigated.

This presents possibilities for model reduction, which can reduce the computational burden and related simulation time considerably. In reservoir simulation literature, a large number of studies on different reduction methods can be found. These methods are either focused on keeping the grid block structure, but reduce the number of required grid blocks [30,9] or are aimed at defining a completely different, more appropriate model structure [27]. Although several methods have been explored, the long-term prediction accuracy of these reduced-order models is as of yet unsatisfactory.

\section{ADDITIONAL OPTIMIZATION OPPOR- TUNITIES}

The closed-loop approach presented in this paper is just the first step towards an on-line, real-life application of a closed-loop reservoir management system. In the road ahead many obstacles still need to be overcome. This provides opportunities for the field of system and control engineering to make a valuable contribution.

The closed-loop management approach, as presented in Section 6, uses production data to reduce uncertainty. However, as the identifiability of parameters is limited, so is the uncertainty reduction. When uncertainty cannot be reduced any further, it should be addressed in the optimization procedure by means of a robust optimization technique. In Van Essen [25], robust optimization was performed using a set of geological realizations, reflecting the range of uncertainty.

The use of NPV as sole objective in the operation of a reservoir over several decades is a rather simplified representation of the objectives pursued in reality. More realistic objectives exhibit both long and short-term aspects. As NPV only addresses the long-term, in optimal life-cycle strategies short-term targets are generally not met. A multiobjective approach is required to improve reservoir management on all relevant layers [24, 21].

In the theory and examples presented in this work, we considered the well configuration (number and location of wells) to be fixed. Finding a good well configuration is however an optimization problem in itself and a number of optimization methods have been studied so far [29, 7].

\section{CONCLUSIONS}

In this paper we have motivated and illustrated that there are challenging opportunities for model-based control and optimization techniques to contribute substantially to the increase of economic efficiency in reservoir engineering. By smartly operating control valves at water injectors and oil producers, the flow conditions in the reservoir can be manipulated so as to optimize oil recovery. Modelbased strategies have been examined in both open-loop and closed-loop forms. A crucial aspect and challenge is the derivation of a reliable model of the permeability structure of the reservoir. Estimating these parameters from data has been illustrated using sequential Bayesian techniques, and proves to be hindered by lack of identifiability. Reparametrization and/or regularization is necessary in order to guarantee sensible results with limited variance. 
On the other hand, while estimated models might seem to have rather poor quality, the subsequent model-based optimization is quite satisfactory. The search for reduced order models - both in terms of state and of parameter complexity - that can be validated from data and that reflect the most important phenomena for control/optimization is a crucial step to be examined. Additional challenges are: the incorporation of model uncertainty (robustness) in the optimization, multi-objective optimization, as well as improving the information content of the measurements by particularly designed experiments.

\section{REFERENCES}

[1] F. Allgöwer and A. Zheng. Nonlinear Model Predictive Control. Birkhäuser, 2000.

[2] K. Aziz and A. Settari. Petroleum Reservoir Simulation. Applied Science Publishers, 1979.

[3] L. T. Biegler. Efficient solution of nonlinear programming and NMPC problems. In F. Allgöwer and A. Zheng, editors, Nonlinear Model Predictive Control, Progress in Systems and Control Theory, pages 219-244. Birkh auser, 2000.

[4] L.T. Biegler and I.E. Grossmann. Retrospective on optimization. Computers \& Chemical Engineering, 28(8):1169-1192, 2004.

[5] D.R. Brouwer, G. Naevdal, J.D. Jansen, E.H. Vefring, and C.P.J.W. van Kruijsdijk. Improved reservoir management through optimal control and continuous model updating. In SPE Annual Technical Conference and Exhibition, Houston, Texas, U.S.A., September 2004. SPE 90149-MS.

[6] A. E. Bryson. Dynamic Optimization. Addison Wesley, Menlo Park, CA, 1999.

[7] D.Y. Ding. Optimization of well placement using evolutionary algorithms. In Europec/EAGE Conference and Exhibition, Rome, Italy, June 2008. SPE 113525-MS

[8] U. Diwekar. Introduction to Applied Optimization, volume 22 of Springer Optimization and Its Applications. Springer, $2^{\text {nd }}$ edition, 2008.

[9] L.J. Durlofsky, R.A. Behrens, R.C. Jones, and A. Bernath. Scale up of heterogeneous three dimensional reservoir descriptions. SPE Journal, 1(3):313-326, September 1996. SPE 30709.

[10] G. Evensen. Sequential data assimilation with a nonlinear quasi-geostrophic model using Monte Carlo methods to forecast error statistics. J. Geophys. Res., 99(C5):101431062, 1994.

[11] G. Evensen. Data Assimilation. Springer, 2007.

[12] M. S. Grewal and K. Glover. Identifiability of linear and nonlinear dynamical systems. IEEE Trans. Automatic Control, 21(6):833-837, 1976.

[13] Y. Gu and D. Oliver. An iterative ensemble Kalman filter for multiphase fluid flow data assimilation. SPE Journal, 12(4):438-446, 2007.

[14] T. Heijn, R. Markovinovic, and J.D. Jansen. Generation of low-order reservoir models using system-theoretical concepts. SPE Journal, 9(2):202-218, June 2004. SPE 88361.

[15] J.D. Jansen, O.H. Bosgra, and P.M.J. Van den Hof. Modelbased control of multiphase flow in subsurface oil reservoirs. Journal of Process Control, 18(9):846-855, 2008.

[16] J.D. Jansen, S.D. Douma, D.R. Brouwer, P.M.J. Van den Hof, O.H. Bosgra, and A.W. Heemink. Closed loop reservoir management. In SPE Reservoir Simulation Symposium, The Woodlands, Texas, U.S.A., February 2009. SPE 119098-MS.
[17] L. Ljung. System Identification: Theory for the User. Prentice-Hall, Englewood Cliffs, NJ, second edition, 1999.

[18] G. Naevdal, L. M. Johnsen, S. I. Aanonsen, and E. H. Vefring. Reservoir monitoring and continuous model updating using ensemble kalman filter. SPE Journal (SPE 84372), 10(1):66-74, 2005

[19] W.F. Ramirez. Application of optimal control theory to enhanced oil recovery. Elsevier, 1987.

[20] D. G. Robertson and J. H. Lee. A least squares formulation for state estimation. J. Process Control, 5(4):291-299, 1995.

[21] L. Saputelli, M. Nikolaou, and M.J. Economides. Selflearning reservoir management. SPE Reservoir Evaluation \& Engineering, 8(6):534-547, December 2005. SPE 84064-PA.

[22] P. Sarma, L.J. Durlofsky, and K. Aziz. Efficient closedloop production optimization under uncertainty. In $S P E$ Europec/EAGE Annual Conference, Madrid, Spain, June 2005. SPE 94241-MS

[23] J. F. M. Van Doren, P. M. J. Van den Hof, J. D. Jansen, and O. H. Bosgra. Determining identifiable parameterizations for large-scale physical models in reservoir engineering. In M.J. Chung, P. Misra, and H. Shim, editors, Proc. 17th IFAC World Congress, pages 11421-11426, Seoul, 611 July 2008.

[24] G.M. van Essen, P.M.J. Van den Hof, and J.D. Jansen. Hierarchical long-term and short-term production optimization. In to be presented at the SPE Annual Technical Conference and Exhibition, New Orleans, Louisiana, U.S.A., October 2009. SPE 124332.

[25] G.M. van Essen, M.J. Zandvliet, P.M.J. Van den Hof, O.H. Bosgra, and J.D. Jansen. Robust waterflooding optimization of multiple geological scenarios. SPE Journal, 14(1):202210, March 2009. SPE 102913-PA.

[26] C. Wang, G. Li, and A.C. Reynolds. Production optimization in closed-loop reservoir management. In SPE Annual Technical Conference and Exhibition, Anaheim, California, U.S.A., November 2007. SPE 109805-MS.

[27] D. Weber, T. F. Edgar, L. W. Lake, L. Lasdon, S. Kawas, and M. Sayarpour. Improvements in capacitance-resistive modeling and optimization of large scale reservoirs. In SPE Western Regional Meeting, San Jose, California, U.S.A., March 2009. SPE 121299-MS.

[28] M. J. Zandvliet, J. F. M. Van Doren, O. H. Bosgra, J. D. Jansen, and P. M. J. Van den Hof. Controllability, observability and identifiability in single-phase porous media flow. Computational Geosciences, 12(4):605-622, December 2008.

[29] M. J. Zandvliet, M. Handels, G. M. van Essen, D. R. Brouwer, and J. D. Jansen. Adjoint-based well-placement optimization under production constraints. SPE Journal, 13(4):392-399, December 2008. SPE 105797-PA.

[30] P. Zhang, G. Pickup, and M. Christie. A new practical method for upscaling in highly heterogeneous reservoir models. SPE Journal, 13(1):68-76, March 2008. SPE 103760-PA. 